\title{
Effects of Combined Aerobic-Strength Training vs Fitness Education Program in COPD Patients
}

\author{
Authors \\ Nicoletta Rinaldo ${ }^{*}$, Elisabetta Bacchi ${ }^{2 *}$, Giuseppe Coratella ${ }^{1,3 *}$, Francesca Vitali ${ }^{1}$, Chiara Milanese ${ }^{1}$, Andrea Rossi $^{4}$, \\ Federico Schena', Massimo Lanza'
}

\section{Affiliations}

1 Department of Neurological, Biomedical and Movement Sciences, University of Verona, Italy

2 Section of Endocrinology, Diabetes and Metabolism, Department of Medicine, University of Verona, Italy

3 Department of Biomedical Sciences for Health, University of Milano, Italy

4 Department of Medicine, Azienda Ospedaliera Universitaria Integrata Verona, Pulmonary Unit, Verona, Italy

\section{Key words}

aerobic training, strength training, chronic obstructive pulmonary disease, quality of life, walking capacity, muscle strength, bone mineral content

accepted 14.05.2017

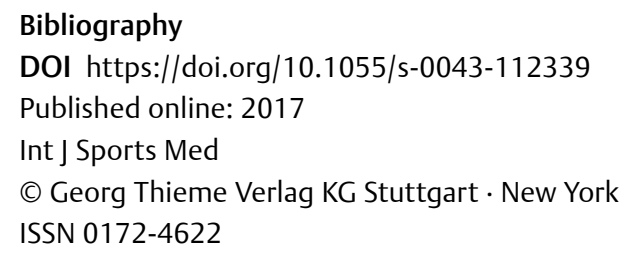

\section{Correspondence}

Dr. Giuseppe Coratella

Department of Neurological, Biomedical and Movement Sciences

University of Verona

via casorati 43
37131, Verona

Italy

Tel.: + 39/347/1948 321, Fax: + 39/045/8425 131

giuseppe.coratella@univr.it

\section{ABSTRACT}

We compared the effects of a new physical activity education program approach (EDU), based on a periodically supervised protocol of different exercise modalities vs traditionally supervised combined strength-endurance training (CT) on healthrelated factors in patients with stable chronic obstructive pulmonary disease (COPD). Twenty-eight COPD patients without comorbidities were randomly assigned to receive either EDU or CT. CT was continuously supervised to combine strength-endurance training; EDU was taught to progressively increase the rate of autonomous physical activity, through different training modalities such as Nordic walking, group classes and circuit training. Body composition, walking capacity, muscle strength, flexibility and balance, total daily energy expenditure and quality of life were evaluated at baseline, after 28 weeks training period ( $3 \mathrm{~d} /$ week) and after a 14-week follow-up. No adverse events occurred during the interventions. After training, CT and EDU similarly improved walking capacity, body composition and quality of life. However, after 14 weeks of follow-up, such improvements were not maintained. Only in CT, muscle strength and flexibility improved after training but returned to baseline after follow-up. EDU, similar to CT, can effectively and safely improve health-related parameters in COPD patients. EDU could be an attractive alternative to traditional supervised training for improving quality of life in COPD patients.

\section{Introduction}

Chronic obstructive pulmonary disease (COPD) is characterized by a progressive airflow limitation that is not fully reversible and is often associated with extra-pulmonary comorbid conditions [33]. Engagement in regular physical activity can provide effective ways to avoid impairments in functional capacity and to prevent physical disability in COPD patients [24, 29, 31]. It follows then that long-

* These authors equally contributed to the work term strategies for promoting and maintaining an active lifestyle are strongly advocated in such patients [19]. Detailed recommendations for pulmonary rehabilitation in COPD patients have documented that physical activity does improve exercise capacity, muscle strength, disease symptoms and quality of life [29]. However, the inclusion of COPD patients in physical exercise programs has proven to be difficult, due to a lack of exercise programs geared toward such patients and their reluctance to continue long-term participation [32]. Indeed, it has been shown that dropout rates of up to $50 \%$ and poor compliance $[12,29]$ may be related to the low 
exercise tolerance and higher fatigability associated with COPD $[4,5]$. Finally, there is no consensus on optimal methods to achieve a long-term adherence to programs for improving health-related factors in stable patients with COPD.

To date, few studies have compared the effects of different approaches to planning physical activity on health-related parameters in stable COPD patients $[12,28]$. Combined exercise training that includes aerobic, strength, balance and flexibility training has been shown to improve clinical and functional capacities and compliance [28]. However because COPD places not only physical limitations but also psychosocial stress on the patients suffering such a condition, it could be interesting to understand the effects of a different approach on the health-related parameters and quality of life in these patients. Particularly, a customized physical activity education program developed and periodically supervised by an exercise specialist may be more appropriately oriented to enhance the participant's autonomy and to encourage COPD patients to take greater responsibility over their own training. Such an education-to-training approach aims to provide support for autonomous motivation to exercise for patients. Such motivation is central to the self-determination theory [11], which was recently integrated into the theory of planned behavior to provide empirical support for studying motivation in health contexts [13]. Research has shown that persistence in health behavior is associated with autonomous forms of motivation [14]. To reach these goals, the protocol of the physical activity education program included explicit instructions, worked examples, and role-play with an accompanying training manual to ensure quality and consistency in the techniques adopted and behaviors displayed. Furthermore, better understanding these aspects may shed light on the effects of a physical activity education program that fosters patient autonomy, can be adapted to participants' needs, and is conducive to building a relationship between the exercise specialist and the COPD patient. To address these issues, we compared the effects of a 28-week physical activity education program with 14 weeks of follow-up vs a supervised combined exercise training on body composition, functional capacity, daily energy expenditure, quality of life, and adherence to training protocols in stable COPD patients. We expect such a customized physical activity education program potentially may benefit health-related parameters similar to the traditional combined training in COPD patients.

\section{Methods}

\section{Design}

This randomized trial was designed to evaluate the feasibility and effectiveness of a physical activity education program as compared with combined exercise training in stable COPD patients. The present study lasted 42 weeks in all. Assessments were performed at baseline, at the end of the interventions (28 weeks), and at 14 weeks of follow-up. The primary outcome was the 6-min walk test (6MWT). Assuming an $\alpha$-error of 0.05 , power of $0.80,2$ groups, 3 repeated measures, an effect size of 0.5 (medium), correlation among repeated measures of 0.8 , and non-sphericity correction of 0.8 , an a-priori power analysis calculation showed that a total sample size of 20 participants was needed (G-Power 3.1, Dusseldorf,
Germany). Because COPD patients are noted to have psychological barriers to participation in exercise training [20] and because dropout rates are known to be high, we aimed for a total sample size of 28 COPD patients. Allocation and randomization were completed by one of the researchers without any contact or knowledge of the participants. The participants were ranked according to their pulmonary capacity (forced expiratory volume). Then, a restricted block (size $=4$ ) randomization, generated by free online software (www.randomization.com) was used to allocate the participants within the 2 groups. Thus, no allocation concealment mechanisms were necessary.

\section{Participants}

Participants ( $n=28$ ) were recruited at the Cardiovascular and Thoracic Department, Respiratory Division, ULSS 20, Verona, Italy, enrolled from January 2013 to January 2014, and followed up until November 2014. Inclusion criteria were: male sex, clinically stable mild-to-severe COPD, and age > 60 years. Exclusion criteria were: unstable cardiac disease, pneumonia, pulmonary embolism, pulmonary vascular disease, respiratory infections, lung cancer, thoracic malignancy, and bone fractures. Participants were included who had not attended a pulmonary rehabilitation program during the previous year. The participants were randomly assigned to receive either physical activity education (EDU, $n=14$ ) or supervised combined exercise training (CT, $n=14)$. Written informed consent was obtained from all participants before the study began. The study was approved by the Ethics Committee of the University of Verona, met the ethical criteria for studies involving human subjects according the Declaration of Helsinki, and meets the ethical standards of the journal [15].

\section{Measures}

\section{Pulmonary function test}

Forced expiratory volume (FEV 1 ) and forced vital capacity (FVC) were measured using a pulmonary function instrument with computer processing (Quark, Cosmed, Rome, Italy) according to the methods of the American Thoracic Society/European Respiratory Society (ATS/ERS) Task Force [23]. The predicted values of FEV ${ }_{1}$ and $\mathrm{FEV}_{1} /$ FVC were calculated according to ATS/ERS [35].

\section{Body composition assessment}

Body mass was measured to the nearest $0.1 \mathrm{~kg}$ (Tanita electronic scale BWB-800 MA, Tanita Corporation, Tokyo, Japan) and stature was measured to the nearest mm (Harpenden stadiometer, Holtain Ltd., Crymych, Pembs. UK). Body-mass index (BMI) was calculated. Whole-body composition (fat mass percentage [FM, \%], lean mass percentage [LM, \%], and bone mineral content [BMC, kg]) was evaluated by means of dual-energy X-ray absorptiometry (DXA) using a total body scanner (QDR Explorer W, Hologic, Marlborough, MA, USA; fan-beam technology, software for Windows XP version 12.6.1) according to the manufacturer's instructions. All scans were performed by a single operator [10].

\section{6-min walk test}

The 6-min walk test (6MWT) was performed according to a standardized protocol [7]. Briefly, participants were instructed to walk on a 30-meter shuttle at the highest feasible speed for $6 \mathrm{~min}$. The 
distance walked in meters at the end of the test was recorded. Participants were allowed to stop and rest during the test. Ratings of perceived exertion (RPE) were measured using the Borg CR10 scale modified for dyspnea [22] at the end of the test.

\section{Strength, flexibility, and balance tests}

Upper body and bilateral lower limb strength (1RM) (chest press and leg extension, Technogym, Cesena, Italy) was assessed separately. The 1 RM was calculated using the Brzycki formula [8]. Each set was separated by 60 s of passive recovery [9]. The sit-and-reach test was performed to assess flexibility of the lower back and hamstring muscles [17]. Briefly, participants were asked to sit on the floor and, with knees straight, place hand over hand and reach forward as far as possible without bending the knees. The best of 3 trials was recorded. A timed one-leg stance test ( $1 \mathrm{~min}$ ) was used to assess postural steadiness in a static position [18]. Participants failed the trial when the contralateral foot touched the ground. The better of 2 separate trials for each limb were summed to calculate the total score.

Assessment of energy expenditure, quality of life, and adherence

Daily total energy expenditure (TEE) was measured with a Sense Wear Armband (SWA, Body Media Inc, Pittsburgh, PA, USA). This wearable armband contains an inertia sensor and is validated as an effective and reliable device to evaluate daily TEE [6], calculated as the daily average over $48 \mathrm{~h}$ monitoring. Quality of life (QoL) was assessed with the Maugeri Respiratory Failure questionnaire (MRF26). This 26 -item questionnaire investigates daily activity, cognitive function, and invalidity in COPD patients. Item scores are summed, with higher scores indicating worse health status [34]. Adherence was defined as attendance recorded at each training session.

\section{Interventions}

The study was carried out at the Fitness Centre of the Exercise and Sport Science School of Verona University. All the supervised training sessions were monitored by exercise specialists.

The EDU program was scheduled as follows:

Weeks 1-5: supervised training sessions 3 days/week; weeks 6-10: supervised training sessions 2 days/week and self-directed training sessions 1 day/week; weeks 10-14: supervised training sessions 1 day/week and self-directed training sessions 2 days/ week; weeks 15-28: self-directed training sessions 3 days/week. Each supervised training session lasted 60 min and included 3 alternating types of physical activity: aerobics classes with flexibility and balance exercises, Nordic walking, or weight-free exercises in circuit training. In more detail, exercises during the aerobic classes were performed at a self-reported intensity from 3 to 4 out of $0-10$ on the CR-10 modified Borg scale, and they included music-based weight-free exercises ( 1 set $x 8$ repetitions, training recovery ratio as 1 to 2/3) tailored for such a population [16]. The Nordic walking session lasted from 10 to 20 min at self-selected speeds, with an RPE ranging from 3 to 4 . Finally, weight-free exercises (e. g.,: squats, lunges, crunches, tailored push-ups, etc.) were performed as circuit training, from 10 to 15 repetitions per exercise and from 2 to 4 circuits, with 4 min recovery between the circuits. We ex- pected that such variety in training routines would promote autonomy, knowledge about different types of exercises, and selfmanagement of exercise-induced symptoms. Participants also received brochures with information about the local physical activity facilities.

The CT program consisted of structured fitness exercises 3 days/ week with at least 1 day in between each session. The training protocol was in line with published recommendations [16]. The participants self-monitored the intensity and duration of endurance and resistance exercises to avoid exertional dyspnea [21]. Briefly, the training protocol combined both aerobic and resistance exercises for a total of $60 \mathrm{~min}$. Endurance exercises consisted of $30 \mathrm{~min}$ of cycling, treadmill walking or using an upper limb ergometer at an intensity from 3 to 4 out of $0-10$ on the CR-10 modified Borg scale. Workload intensity was adjusted as a function of RPE. Resistance exercises consisted of 4 sets of lower limb (leg press and knee extension), upper limb (chest press and shoulder press), and trunk (free-weight abdominal crunch and back extension) exercises performed between 50 and $80 \%$ of 1RM. Load intensity was adjusted every 3 to 4 weeks according to 1RM test results. Each training session ended with flexibility and balance exercises using proprioceptive boards. All participants were instructed to continue with their prescribed exercise program during the 14-week follow-up period.

\section{Statistical analysis}

Statistical analysis was performed using IBM SPSS Statistics version 20 (IBM Corp, Armonk, NY, USA). Data normality was evaluated using the Kolmogorov-Smirnov test. Data sphericity was tested using Mauchly's test. Repeated measures ANOVA was used to compare variables measured over time as the dependent variable and the effects for time, study group, and time $x$ group interaction. When time $x$ group interactions results were significant, post-hoc analysis with Bonferroni's correction was performed. Data are expressed as mean \pm standard deviation (SD). Significance was set at $\mathrm{p}<0.05$.

\section{Results}

Of the 269 male COPD patients initially contacted, 28 volunteered to participate and 24 completed the study ( $\vee$ Fig. 1). 4 participants dropped out for reasons unrelated to the study. The data from 24 participants (EDU group, $\mathrm{n}=12$ and CT group, $\mathrm{n}=12$ ) were used in the analysis. All variables resulted in normal distribution. - Table 1 presents the baseline characteristics of the participants.

Training adherence, as measured by attendance at sessions, was $100 \%$ and $87 \%$, in the EDU and the CT group, respectively. Changes in values on the 6MWT, muscle strength, flexibility and balance tests are shown in $>$ Table 2 . Differences between pre- and posttraining values on the chest press (1RM), leg extension (1RM), and sit-and-reach test were significant only for the CT group. However, the values for these parameters significantly worsened at the 14week follow-up assessment. Improvements in post-training 6MWT, RPE, and balance test values over baseline were observed in both groups but they were not maintained for the 6MWT and balance test at follow-up, when the values for these parameters were significantly reduced. 


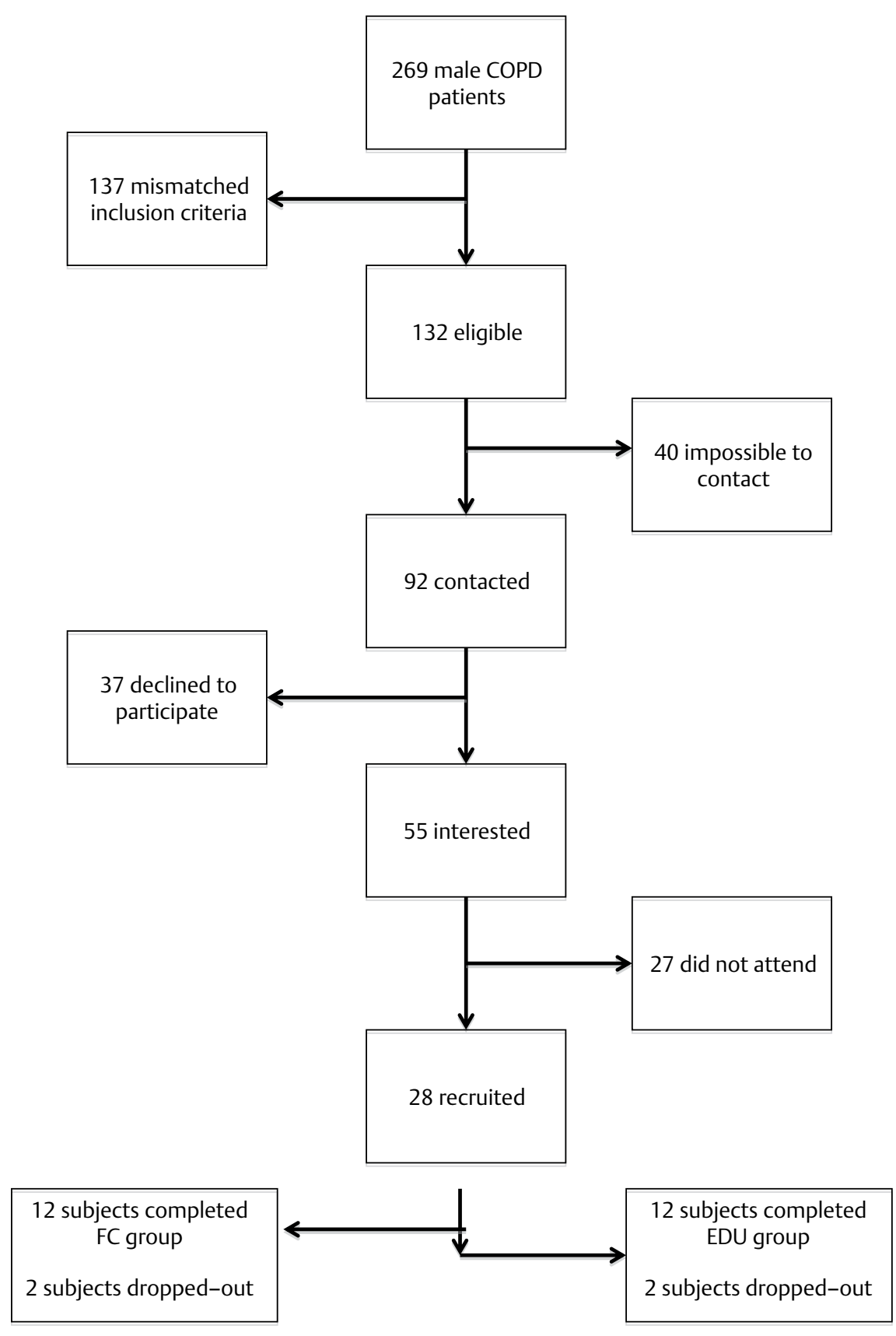

-Fig. 1 Flow diagram of the recruitment trial.

- Table 3 presents the changes in body composition parameters. The BMC in both groups was significantly higher after training, while FM\% and LM\% remained unchanged between baseline and post-training assessment. Interestingly, FM and LM were worse, albeit slightly, at follow-up assessment.

Changes in TEE and QoL parameters are shown in > Table 4. TEE remained unchanged after the training period, while they were significantly reduced at follow-up in both groups. The significant improvements in QoL that resulted after the training period in both groups were lost at follow-up.

\section{Discussion}

This is the first randomized trial designed to explore the effectiveness of a new physical activity education program, based on a periodically supervised protocol of different exercise modalities vs. traditional supervised combined exercise training. In line with our hypothesis, improvements in 6MWT, balance, BMC, and QoL were observed in both groups after 28 weeks of training. However, at follow-up assessment 14 weeks later the values for body composition, 6MWT, balance, TEE, and QoL were all impaired. Finally, only 
> Table 1 Demographics and clinical characteristics (mean \pm SD) at baseline.

\begin{tabular}{|l|c|c|c|c|c|}
\hline & Age (yrs) & FEV1 (\%predicted) & FEV1/FVC (\%predicted) & BMI $\left[\mathbf{k g} \cdot\left(\mathbf{m}^{2}\right)^{-\mathbf{1}}\right]$ & BODE \\
\hline CT group & $66.2 \pm 4.2$ & $60.1 \pm 24.3$ & $53.7 \pm 17.7$ & $28.4 \pm 5.7$ & $3.00 \pm 0.85$ \\
\hline EDU group & $66.1 \pm 4.5$ & $72.2 \pm 18.8$ & $58.3 \pm 13.8$ & $29.9 \pm 4.4$ & $3.18 \pm 1.32$ \\
\hline
\end{tabular}

CT denotes combined training, EDU physical activity education, FEV1 forced expiratory volume, FEV1/FVC ratio of forced expiratory volume to forced vital capacity, BMI body-mass index, BODE index Body-mass index, airflow Obstruction, Dyspnea, and Exercise.

- Table 2 Performance parameters measured at baseline, at 28 weeks, and at 14 weeks of follow-up.

\begin{tabular}{|c|c|c|c|c|c|c|}
\hline & Baseline & 28 weeks & Follow-up & Factor time & $\begin{array}{l}\text { Factor } \\
\text { group }\end{array}$ & $\begin{array}{c}\text { Time } x \text { group } \\
\text { interaction }\end{array}$ \\
\hline $6 \mathrm{MWT}(\mathrm{m})$ & & $*$ & $\#$ & $p=0.009$ & $p=0.094$ & $p=0.565$ \\
\hline $\mathrm{CT}$ & $455 \pm 110$ & $480 \pm 121$ & $409 \pm 172$ & & & \\
\hline EDU & $519 \pm 72$ & $540 \pm 48$ & $506 \pm 65$ & & & \\
\hline RPE $(0-10)$ & & $*$ & & $\mathrm{p}=0.019$ & $p=0.731$ & $p=0.129$ \\
\hline CT & $6.3 \pm 0.9$ & $5.7 \pm 0.9$ & $5.7 \pm 1.0$ & & & \\
\hline EDU & $6.4 \pm 0.9$ & $5.6 \pm 1.1$ & $5.7 \pm 1.0$ & & & \\
\hline Leg extension (kg) & & & & $p=0.005$ & $p=0.197$ & $p=0.046$ \\
\hline CT & $59.2 \pm 18.2$ & $65.8 \pm 24.0 \S$ & $54.6 \pm 26.5 \$$ & & & \\
\hline EDU & $64.1 \pm 21.8$ & $65.6 \pm 20.4$ & $60.2 \pm 17.3$ & & & \\
\hline Chest press $(\mathrm{kg})$ & & & & $p=0.000$ & $p=0.028$ & $p=0.011$ \\
\hline CT & $41.1 \pm 8.9$ & $50.0 \pm 8.8 \S$ & $39.5 \pm 7.4 \$$ & & & \\
\hline EDU & $57.8 \pm 15.1$ & $54.3 \pm 13.9$ & $49.6 \pm 9.2$ & & & \\
\hline Flexibility $(\mathrm{cm})$ & & & & $p=0.001$ & $p=0.565$ & $p=0.033$ \\
\hline CT & $-11.8 \pm 12.0$ & $-5.3 \pm 10.5 \S$ & $-9.7 \pm 12.2 \$$ & & & \\
\hline EDU & $-13.5 \pm 13.9$ & $-10.7 \pm 14.6$ & $-11.6 \pm 12.7$ & & & \\
\hline Balance (s) & & * & ${ }^{*} \#$ & $p=0.000$ & $p=0.482$ & $p=0.299$ \\
\hline CT & $47.7 \pm 48.2$ & $107.2 \pm 22.3$ & $81.4 \pm 41.7$ & & & \\
\hline EDU & $72.5 \pm 47.6$ & $104.6 \pm 33.8$ & $89.6 \pm 36.3$ & & & \\
\hline
\end{tabular}

- Table 3 Body composition parameters measured at baseline, at 28 weeks of training, and at 14 weeks of follow-up.

\begin{tabular}{|c|c|c|c|c|c|c|}
\hline & Baseline & 28 weeks & Follow-up & Factor time & Factor group & $\begin{array}{c}\text { Time } \mathrm{x} \text { group } \\
\text { interaction }\end{array}$ \\
\hline BMC (kg) & & * & & $p=0.002$ & $p=0.038$ & $p=0.320$ \\
\hline CT & $2.19 \pm 0.39$ & $2.23 \pm 0.40$ & $2.20 \pm 0.41$ & & & \\
\hline EDU & $2.60 \pm 0.46$ & $2.62 \pm 0.48$ & $2.61 \pm 0.47$ & & & \\
\hline FM (\%) & & & $\#$ & $p=0.015$ & $p=0.760$ & $p=0.837$ \\
\hline CT & $29.2 \pm 5.0$ & $29.2 \pm 4.7$ & $30.0 \pm 4.5$ & & & \\
\hline EDU & $29.0 \pm 4.9$ & $28.6 \pm 4.6$ & $29.6 \pm 4.2$ & & & \\
\hline LM (\%) & & & \# & $p=0.025$ & $p=0.938$ & $p=0.839$ \\
\hline $\mathrm{CT}$ & $67.9 \pm 4.9$ & $67.9 \pm 4.6$ & $67.2 \pm 4.4$ & & & \\
\hline EDU & $67.8 \pm 4.8$ & $68.2 \pm 4.5$ & $67.3 \pm 4.2$ & & & \\
\hline
\end{tabular}

Data are means \pm SD. ${ }^{*} \mathrm{p}<0.05$ comparing pooled data to baseline; $\# \mathrm{p}<0.05$ comparing pooled data to 28 weeks. CT denotes combined training group, EDU physical activity education group, BMC bone mineral content, FM fat mass, LM lean mass.

CT increased muscle strength after the training period, even though such improvements returned to baseline after the follow-up.

The novel findings of this study are that marked improvement was achieved in several health-related parameters at assessment on completion of the EDU and CT programs. QoL and walking ca- pacity are important health aspects for COPD patients and aerobic or combined exercise training are known to improve both [29]. Our results show that an EDU program, when delivered by an exercise specialist, can be a feasible and effective approach to improving autonomy, QoL, and aerobic capacity in patients with stable COPD. 
- Table 4 Total energy expenditure and quality of life measured at baseline, at 28 weeks of training, and at 14 weeks of follow-up.

\begin{tabular}{|c|c|c|c|c|c|c|}
\hline & Baseline & 28 weeks & Follow-up & Factor time & Factor group & $\begin{array}{c}\text { Time } \mathrm{x} \text { group } \\
\text { interaction }\end{array}$ \\
\hline TEE (kcal) & & & \# & $p=0.049$ & $p=0.441$ & $p=0.852$ \\
\hline CT & $3070 \pm 475$ & $3140 \pm 522$ & $2890 \pm 617$ & & & \\
\hline EDU & $3465 \pm 580$ & $3660 \pm 974$ & $3290 \pm 654$ & & & \\
\hline QoL (A.U.) & & * & \# & $p=0.000$ & $p=0.063$ & $p=0.096$ \\
\hline CT group & $7.2 \pm 3.8$ & $4.1 \pm 4.4$ & $6.1 \pm 4.3$ & & & \\
\hline EDU group & $5.3 \pm 2.1$ & $2.8 \pm 2.7$ & $4.1 \pm 2.1$ & & & \\
\hline
\end{tabular}

Data are means \pm SD. ${ }^{*} p<0.05$ comparing pooled data to baseline; $\# p<0.05$ comparing pooled data to 28 weeks. CT denotes combined training group, EDU physical activity education group, TEE total energy expenditure, QoL quality of life.

Consistent with previous studies $[3,25,26]$, no adverse events occurred in the present study, indicating that EDU and CT are safe protocols for stable COPD patients.

In the present study few differences were found between the groups. In particular, we noted that muscle strength and flexibility significantly improved after intervention and decreased at followup assessment only in the CT group. This difference may have been due to the greater amount of endurance training the EDU group received. To the best of our knowledge, few studies have compared combined training with aerobic plus resistance training with other physical activity programs in COPD patients. As compared with other types of exercise, greater improvements in muscle strength were noted after combined exercise training or strength training $[3,25,26,28]$.

EDU and CT are appropriate interventions to improve several health-related parameters; however, some improvements gained during training were lost at follow-up assessment. Furthermore, body composition and TEE values were impaired in both groups at follow-up, which suggests that periodic or continuous supervised training should be recommended to ensure that COPD patients maintain the health benefits gained and continue to engage in regular physical activity.

In particular, no significant improvement in daily TEE was observed in either the EDU or the CT group and the TEE values were decreased in both groups at follow-up assessment. A recent report indicated an improvement in functional status but no increase in TEE in COPD patients after 12 weeks of supervised combined training or calisthenics exercise programs [28]. Similarly, another study found that walking time improved only after 6 months, but not after 3 months, of rehabilitation [27]. The lack of an increase in TEE may be explained in part by the program duration, the type of exercises, and the health status of the COPD patients, while the decrease in daily TEE at follow-up might have been related to the lack of supervised exercise.

Interestingly, an increase in FM and a decrease in LM were observed at follow-up. Contrary to our results, 2 studies found increases in muscle mass after combined training as compared with aerobic exercise in COPD patients [3, 26]. Unfortunately, the use of different measurement techniques and study designs (e. g., computed tomography or DXA) does not allow for appropriate comparisons. What the present data do suggest, however, is that both interventions are effective in avoiding decreases in fat free mass and increases in fat mass. In addition, BMC was improved in both groups, though the underlying mechanisms are not fully understood in COPD.

Emerging literature indicates that the reduced balance ability in older adults with COPD increases the risk of falling [30]. The few studies that have investigated the effects of balance training in COPD patients as part of a rehabilitation program have produced inconsistent results [1, 2]. In our study, balance control was markedly improved in both groups at 28-week assessment but was decreased at follow-up, suggesting that a long-term, structured exercise program may help to maintain this important functional capacity.

The main methodological limitations of our study are the lack of a control group and the somewhat small sample size, which, however, did not preclude the observation of significant differences over time. In addition, only male patients were recruited. Although it does exclude any possible sex-related confounding factors, it would be interesting to evaluate such protocols in female participants. Notwithstanding these limitations, the main strengths of the present study are its randomized controlled design, a new physical activity education approach, the duration of the study, including the lengthy follow-up period, and the measurement of several health-related parameters. In this context, the collaboration with exercise specialists was key to achieving the potential benefits of combined training and physical activity education.

In summary, our results demonstrated that both EDU and CT similarly improved walking capacity, balance, BMC, QoL, and adherence to training in stable COPD patients, although such parameters returned to baseline after 14 weeks of follow-up. In addition, muscle strength improved after training only in CT and, similar to the above-mentioned parameters, it returned to baseline after the follow-up. Remarkably, we have shown that this new physical activity approach, based on active education-to-training through different exercise experiences in COPD patients, may have overlapping results compared to a traditional supervised combined strength-endurance training protocol. Therefore, clinicians and exercise specialists may consider it a convenient alternative to combined training in order to improve health-related parameters in stable COPD patients. 


\section{Acknowledgements}

The authors received no financial support. We thank all the participants in this study and the staff of the Exercise and Sport Science School, University of Verona, for their technical support.

\section{Conflict of interest}

The authors have no conflict to declare.

\section{References}

[1] Beauchamp MK, Janaudis-Ferreira T, Parreira V, Romano JM, Woon L, Goldstein RS, Brooks D. A randomized controlled trial of balance training during pulmonary rehabilitation for individuals with COPD. Chest 2013; 144: 1803-1810

[2] Beauchamp MK, O'Hoski S, Goldstein RS, Brooks D. Effect of pulmonary rehabilitation on balance in persons with chronic obstructive pulmonary disease. Arch Phys Med Rehabil 2010; 91: 1460-1465

[3] Bernard S, Whittom F, Leblanc P, Jobin J, Belleau R, Bérubé C, Carrier G, Maltais $F$. Aerobic and strength training in patients with chronic obstructive pulmonary disease. Am J Respir Crit Care Med 1999; 159: 896-901

[4] Boccia G, Coratella G, Dardanello D, Rinaldo N, Lanza M, Schena F, Rainoldi A. Severe COPD alters muscle fiber conduction velocity during knee extensors fatiguing contraction. COPD J Chronic Obstr Pulm Dis 2016; 2555: 1-6

[5] Boccia G, Dardanello D, Rinaldo N, Coratella G, Schena F, Rainoldi A. Electromyographic manifestations of fatigue correlate with pulmonary function, 6-minute walk test, and time to exhaustion in COPD. Respir Care 2015; 1295-1302

[6] Brazeau A-S, Beaudoin N, Bélisle V, Messier V, Karelis AD, RabasaLhoret R. Validation and reliability of two activity monitors for energy expenditure assessment. J Sci Med Sport 2014

[7] Brooks D, Solway S, Gibbons WJ. ATS statement on six-minute walk test. Am J Respir Crit Care Med 2003; 167: 1287

[8] Brzycki M. Strength testing-predicting a one-rep max from reps-to-fatigue. J Physical Educ Recreat Danc 1993; 68: 88-90

[9] Coratella G, Milanese C, Schena F. Unilateral eccentric resistance training: a direct comparison between isokinetic and dynamic constant external resistance modalities. Eur J Sport Sci 2015; 15: $720-726$

[10] Coratella G, Milanese C, Schena F. Cross-education effect after unilateral eccentric-only isokinetic vs dynamic constant external resistance training. Sport Sci Health 2015; 11: 329-335

[11] Deci EL, Ryan RM. Intrinsic Motivation and Self-Determination in Human Behavior. New York: Springer Science + Business Media; 1985

[12] Greulich T, Kehr K, Nell C, Koepke J, Haid D, Koehler U, Koehler K, Filipovic S, Kenn K, Vogelmeier C, Koczulla AR. A randomized clinical trial to assess the influence of a three months training program (gym-based individualized vs. calisthenics-based non-individualized) in COPD patients. Respir Res 2014; 15: 36

[13] Hagger MS, Chatzisarantis NLD. Integrating the theory of planned behaviour and self-determination theory in health behaviour: $\mathrm{A}$ meta-analysis. Br J Health Psychol 2009; 14: 275-302
[14] Hagger MS, Chatzisarantis NLD, Biddle SJH. The influence of autonomous and controlling motives on physical activity intentions within the Theory of Planned Behaviour. Br J Health Psychol 2002; 7 : 283-297

[15] Harriss D], Atkinson G. Ethical standards in sport and exercise scienceresearch: 2016 update. Int J Sports Med 2015; 36: 1121-1124

[16] lepsen UW, Jørgensen KJ, Ringbæk T, Hansen H, Skrubbeltrang C, Lange $\mathrm{P}$. A combination of resistance and endurance training increases leg muscle strength in COPD: An evidence-based recommendation based on systematic review with meta-analyses. Chron Respir Dis 2015; 12: 132-145

[17] Jones C], Rikli RE, Max J, Noffal G. The reliability and validity of a chair sit-and-reach test as a measure of hamstring flexibility in older adults. Res Q Exerc Sport 1998; 69: 338-343

[18] Jonsson E, Seiger A, Hirschfeld H. One-leg stance in healthy young and elderly adults: a measure of postural steadiness? Clin Biomech 2004; 19: 688-694

[19] Kahn EB, Ramsey LT, Brownson RC, Heath GW, Howze EH, Powell KE, Stone EJ, Rajab MW, Corso P. The effectiveness of interventions to increase physical activity. A systematic review. Am J Prev Med 2002; 22: $73-107$

[20] Keating A, Lee A, Holland AE. What prevents people with chronic obstructive pulmonary disease from attending pulmonary rehabilitation? A systematic review. Chron Respir Dis 2011; 8: 89-99

[21] Langer D, Hendriks E, Burtin C, Probst V, van der Schans C, Paterson W, Verhoef-de Wijk M, Straver R, Klaassen M, Troosters T, Decramer M, Ninane V, Delguste P, Muris J, Gosselink R. A clinical practice guideline for physiotherapists treating patients with chronic obstructive pulmonary disease based on a systematic review of available evidence. Clin Rehabil 2009; 23: 445-462

[22] Mahler DA. The measurement of dyspnea during exercise in patients with lung disease. Chest 1992; 101: 242S-247S

[23] Miller MR, Hankinson J, Brusasco V, Burgos F, Casaburi R, Coates A, Crapo R, Enright P, van der Grinten CPM, Gustafsson P, Jensen R, Johnson DC, MacIntyre N, McKay R, Navajas D, Pedersen OF, Pellegrino R, Viegi G, Wanger J. Standardisation of spirometry. Eur Respir J 2005; 26: 319-338

[24] Nici L, Donner C, Wouters E, Zuwallack R, Ambrosino N, Bourbeau ], Carone M, Celli B, Engelen M, Fahy B, Garvey C, Goldstein R, Gosselink R, Lareau S, Maclntyre N, Maltais F, Morgan M, O’Donnell D, Prefault C, Reardon J, Rochester C, Schols A, Singh S, Troosters T. American Thoracic Society/European Respiratory Society statement on pulmonary rehabilitation. Am J Respir Crit Care Med 2006; 173: 1390-1413

[25] Ortega F, Toral J, Cejudo P, Villagomez R, Sánchez H, Castillo ], Montemayor T. Comparison of effects of strength and endurance training in patients with chronic obstructive pulmonary disease. Am J Respir Crit Care Med 2002; 166: 669-674

[26] Panton LB, Golden J, Broeder CE, Browder KD, Cestaro-Seifer DJ, Seifer FD. The effects of resistance training on functional outcomes in patients with chronic obstructive pulmonary disease. Eur ] Appl Physiol 2004; 91: 443-449

[27] Pitta F, Troosters T, Probst VS, Langer D, Decramer M, Gosselink R. Are patients with COPD more active after pulmonary rehabilitation? Chest 2008; 134: 273-280

[28] Probst VS, Kovelis D, Hernandes NA, Camillo CA, Cavalheri V, Pitta F. Effects of 2 exercise training programs on physical activity in daily life in patients with COPD. Respir Care 2011; 56: 1799-1807

[29] Ries AL, Bauldoff GS, Carlin BW, Casaburi R, Emery CF, Mahler DA, Make B, Rochester CL, Zuwallack R, Herrerias C. Pulmonary Rehabilitation: Joint ACCP/AACVPR Evidence-Based Clinical Practice Guidelines. Chest 2007; 131: 4S-42S 
[30] Roig M, Eng J], Maclntyre DL, Road JD, FitzGerald JM, Burns ], Reid WD. Falls in people with chronic obstructive pulmonary disease: An observational cohort study. Respir Med 2011; 105: 461-469

[31] Spruit MA, Troosters T, Trappenburg JCA, Decramer M, Gosselink R. Exercise training during rehabilitation of patients with COPD: A current perspective. Patient Educ Couns 2004; 52: 243-248

[32] Valero C, Monteagudo M, Llagostera M, Bayona X, Granollers S, Acedo M, Ferro JJ, Rodríguez-Latre L, Almeda J, Muñoz L. Evaluation of a combined strategy directed towards health-care professionals and patients with chronic obstructive pulmonary disease (COPD): Information and health education feedback for improving clinical monitoring and quality-of-life. BMC Public Health 2009; 9: 442
[33] Vestbo J, Hurd SS, Agustí AG, Jones PW, Vogelmeier C, Anzueto A, Barnes PJ, Fabbri LM, Martinez FJ, Nishimura M, Stockley RA, Sin DD, Rodriguez-Roisin R. Global strategy for the diagnosis, management, and prevention of chronic obstructive pulmonary disease: GOLD executive summary. Am J Respir Crit Care Med 2013; 187: 347-365

[34] Vidotto G, Carone M, Jones PW, Salini S, Bertolotti G. Maugeri Respiratory Failure questionnaire reduced form: A method for improving the questionnaire using the Rasch model. Disabil Rehabil 2007; 29: 991-998

[35] Skeletal muscle dysfunction in chronic obstructive pulmonary disease. A statement of the American Thoracic Society and European Respiratory Society. Am J Respir Crit Care Med 1999; 159: S1-S40 